\title{
Estimation de la dénaturation des protéines par spectrofluorimétrie d'absorption moléculaire : applications aux lactosérums industriels
}

\author{
F Saulnier 1, 2, F Ferrero 1, 2, A Choukri 1, \\ JM Girardet 1, G Linden 1 \\ 1 Laboratoire de biochimie appliquée associé à I'INRA-Université de Nancy 1, Faculté des Sciences, \\ BP 239, 54506 Vandœuvre-lès-Nancy Cedex; \\ ${ }^{2}$ Lacto Sérum France, Baleycourt, BP 64, 55102 Verdun Cedex, France
}

(Reçu le 13 février 1991; accepté le 6 mai 1991)

\begin{abstract}
Résumé - La spectrofluorimétrie d'absorption moléculaire avec l'utilisation d'un chromophore comme le 1-anilino-8-naphtalène sulfonate (ANS) permet d'estimer la dénaturation protéique dans les lactosérums de fromagerie. La première partie du travail a consisté à adapter cette technique aux lactosérums, afin d'obtenir une méthode rapide et fiable. La mesure de la densité optique à $280 \mathrm{~nm}$ suffit pour standardiser la concentration en protéines des échantillons et réduire les temps d'analyse. De cette étude, il ressort que la dénaturation protéique est conditionnée par le passé fromager des lactosérums; c'est-à-dire, pour l'essentiel, des traitements thermiques que le lait a subis. Par déplisssement des protéines, les traitements thermiques rendent plus de sites hydrophobes accessibles à l'ANS, ce qui se traduit par une augmentation de fluorescence.
\end{abstract}

spectrofluorimétrie / lactosérum / dénaturation / protéine

Summary - Estimation of protein denaturation by spectrofluorimetry: application to industrial wheys. Molecular absorption spectrofluorimetry using a chromophore such as 1-anilino-8naphthalene sulphonate (ANS) permits the estimation of protein denaturation in industrial whey. In order to obtain a rapid and reliable analysis, protein concentration determination was first necessary. The measurement of absorbance at $280 \mathrm{~nm}$ is sufficient to standardize sample protein concentration and reduce analysis time. The result of this study indicates that protein denaturation depends on process treatments during cheese making, particularly milk heat treatments. Protein unfolding caused by heat treatment renders the hydrophobic sites more available to ANS, which explains the increase in fluorescence.

spectrofluorimetry / whey / denaturation / protein 


\section{INTRODUCTION}

La fluorescence moléculaire s'applique aussi bien aux éléments minéraux qu'aux substances organiques. Des centaines de composés sont fluorescents, et il est en outre possible de rendre fluorescent, par réaction chimique, un composé qui ne l'est pas.

Si cette technique est plus délicate à pratiquer que la spectrométrie d'absorption moléculaire, elle permet cependant en général d'améliorer la sensibilité d'un dosage.

Parmi les différentes applications analytiques qui se sont développées ces dernières années, il convient de citer l'emploi de réactifs chimiques susceptibles de se combiner aux protéines pour les rendre fluorescentes.

L'acide cis-parinarique est utilisé dans de nombreux domaines, en particulier celui des protéines d'origine végétale (Nakai et al, 1980), ou d'origine animale, telle que la $\beta$-lactoglobuline (Waniska et Kinsella, 1984), le lysozyme et l'ovalbumine (Kato et al, 1986), ainsi que l'étude de la texture du yaourt par l'association des protéines de lactosérum avec la micelle de caséine sous l'effet d'une dénaturation thermique (Mottar et al, 1989). Mais cette molécule, qui comporte trois doubles liaisons sur sa chaîne carbonée, est très instable et donc peu pratique à utiliser.

Le 1-anilino-8-naphthalène sulfonate (ANS) est beaucoup plus stable, et il est employé dans le domaine des protéines végétales (Paulson et Tung, 1987) et dans celui des protéines animales comme la $\beta$ lactoglobuline (Mills et Creamer, 1975; Halpin et Richardson, 1985), l'ovalbumine (Hayakawa et Nakai, 1985), la caséine humaine (Slattery et al, 1989). L'emploi de I'ANS avec les protéines laitières permet d'évaluer la dénaturation thermique de dif- férents laits commerciaux (Bonomi et al, 1988; Pagliarini et al, 1990) ou bien le suivi de la coagulation par la présure à différents pH (Peri et al, 1990).,

L'objet de cet article est de corroborer certains résultats obtenus par les précédents auteurs (Bonomi et al, 1988; Pagliarini et al, 1990) et de montrer en particulier que cette méthode est parfaitement adaptée pour évaluer le taux de dénaturation thermique des protéines de lactosérums de fromageries.

Comme l'état plus ou moins dénaturé de ces protéines rejaillit sur la valorisation des lactosérums, nous avons essayé de montrer que la spectrofluorimétrie permet d'obtenir de précieuses indications sur l'origine des lactosérums et sur les traitements thermiques qu'ils ont subis.

\section{MATÉRIEL ET MÉTHODES}

\section{Produits et réactifs}

Les protéines purifiées telles que la $\beta$ lactoglobuline ( $\beta L G)$, l' $\alpha$-lactalbumine $(\alpha L A)$, la sérumalbumine bovine (BSA) et les immunoglobulines G (IgG) proviennent de chez Sigma Chemical Co (St-Louis, États-Unis).

L'ANS est fournie par Serva Feinbiochemica GMBH Co (Heidelberg, Allemagne).

\section{Lactosérums étudiés}

Les lactosérums doux sont concentrés de 6 à $30 \%$ d'extrait sec (ES) sur les sites de production fromagère. Suivant leurs origines et la valeur du $\mathrm{pH}$, ils sont triés et stockés à $30 \%$ d'ES, à une température inférieure à $10^{\circ} \mathrm{C}$. Les lactosérums préconcentrés doux proviennent de la SA Lacto Sérum France (Verdun), et ont des valeurs moyennes de $\mathrm{pH}$ comprises entre 5,80 et 6,20 . 
Les lactosérums acides $(\mathrm{pH}$ moyen de 4,60$)$ sont issus de technologies au lait cru (pâtes molles $A O C$ ), de technologies pâtes fraîches industrielles (procédé par centrifugation). De plus, nous avons étudié divers lactosérums acides de caséinerie préconcentrés à $30 \%$ d'ES, collectés par la SA Lacto Sérum France.

\section{Détermination de la teneur en protéines}

Les lactosérums préconcentrés sont dilués au $1 / 50$ avec du tampon phosphate de potassium

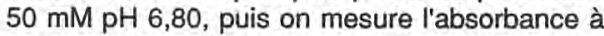
$280 \mathrm{~nm}\left(\mathrm{DO}_{280}\right)$ au spectromètre d'absorption moléculaire (Shimadzu MPS 2000). La corrélation avec la méthode Kjeldahl (Norme FIL N²0) donne l'équation suivante:

$\%$ protéines $(\mathrm{g} / 100 \mathrm{~g})=0,07849 \mathrm{DO}_{280}+0,01476$ avec $r=0,991, n=30$

Ces échantillons, (dilution au 1/50), sont ajustés avec le tampon phosphate de potassium $50 \mathrm{mM} \mathrm{pH} \mathrm{6,80,} \mathrm{à} \mathrm{une} \mathrm{valeur} \mathrm{de} \mathrm{DO}_{280}$ égale à 0,400 .

Dans notre dosage, nous considérons le coefficient d'extinction moléculaire $(\varepsilon)$ identique pour tous nos lactosérums. En effet, $\varepsilon$ dépend du $\mathrm{pH}$ (égal à 6,80 et constant dans notre étude) et de la composition relative en protéines, reflétée par le rapport $\beta L G / \alpha L A$. Ce rapport, déterminé par densitométrie à partir d'un profil électrophorétique en gel de polyacrylamide non dénaturant (Pâquet et al, 1988), a une valeur constante de 2,20 ( $\pm 0,02)$ pour tous les lactosérums étudiés.

\section{Mesure de I'hydrophobicité de surface}

Nous avons adapté aux lactosérums la méthode citée par Bonomi et al (1988). Un multispectre a permis de déterminer les longueurs d'ondes d'excitation $(E X)$ et d'émission (EM) optimales du complexe ANS-protéine. Les essais ont été réalisés avec un spectrofluorimètre Shimadzu RF 5000.

Les longueurs d'ondes retenues sont:

$E X=280 \mathrm{~nm}$

\section{$E M=460 \mathrm{~nm}$}

La bande passante pour chacune des longueurs d'ondes est de $20 \mathrm{~nm}$.

Afin d'éviter tout effet dénaturant du fluorophore, la lecture de la fluorescence est effectuée immédiatement après l'ajout de l'ANS. Audelà de $4 \mathrm{~min}$, nous observons une augmentation de la fluorescence, suite à un déplissement protéique.

La mesure de la cinétique de fixation de I'ANS (préparation à $2,78 \mathrm{mM}$ dans le tampon phosphate de potassium) donne une courbe du type hyperbole équilatère, passant par l'origine des coordonnées. Après linéarisation, nous obtenons 3 paramètres clés :

$F_{\max }$ : Fluorescence maximale, exprimée en unité arbitraire.

$K_{f}$ : Constante de dissociation du complexe ANS-protéines, exprimée en $\mu \mathrm{M}$.

Cette valeur est l'inverse de l'affinité apparente de l'ANS pour les protéines. La valeur de ce paramètre dépend de la densité, du type et de la position des sites hydrophobes.

Le rapport $F_{\max } / K_{\mathrm{f}}$ traduit le nombre et l'affinité des sites hydrophobes de surface (hydrophobicité effective).

L'équation de la droite $1 /$ fluorescence $=$ $f(1 /$ ANS ) permet de déterminer les paramètres de fluorescence. $K_{\mathrm{f}} / F_{\max }$ représente la pente de la droite et $1 / F_{\max }$ est l'ordonnée à l'origine.

\section{RÉSULTATS ET DISCUSSION}

Nous avons fait varier la concentration en protéines de manière à obtenir une $\mathrm{DO}_{280}$ comprise entre 0,1 et 0,4 , et nous avons mesuré la fluorescence maximale atteinte pour ces différentes concentrations. Nous obtenons une droite de corrélation :

$\mathrm{DO}_{280}=4 \times 10^{-3} F_{\max }-73,85 \times 10^{-3}$, avec $r=0,98, n=30$

La fluorescence maximale est donc proportionnelle à la concentration en protéines. 
Mesure de l'hydrophobicité de surface
sur des protéines purifiées

Les mesures sont réalisées avec des solutions protéiques à $4,5 \mathrm{mg} / \mathrm{l}$ dans du tampon phosphate de potassium $50 \mathrm{mM}, \mathrm{pH}$ 6,80 .

La fluorescence en fonction de doses croissantes d'ANS est représentée sur la figure 1. Les différents paramètres obtenus d'après ces courbes sont résumés dans le tableau I. Nous notons d'importantes variations de fluorescence maximale allant de 5,9 (IgG) à 4866 (BSA). Ces variations sont en accord avec celles déjà observées sur les protéines globulaires ( $\beta L G, \alpha L A, B S A$ ) par Shimizu et al (1985) lorsqu'ils utilisent I'ANS. Cependant, selon ces auteurs, les techniques de mesure d'hydrophobicité ne sont pas corrélées entre elles. En effet, un résultat inverse est observé pour la $\beta L G$ et la BSA quand ils utilisent l'acide cis-parinarique. Ils distinguent donc I'hydrophobicité aromatique et l'hydrophobicité aliphatique. De plus, I'hydrophobicité de surface de l' $\alpha$ LA obtenue par Shimizu et al (1985) avec l'ANS comme fluorophore est plus faible que celle que nous observons. En effet, le $K_{\mathrm{f}}$ de cette protéine peut être multiplié d'un facteur 10 si cette dernière fixe des ions bivalents (Musci et Berliner, 1985).

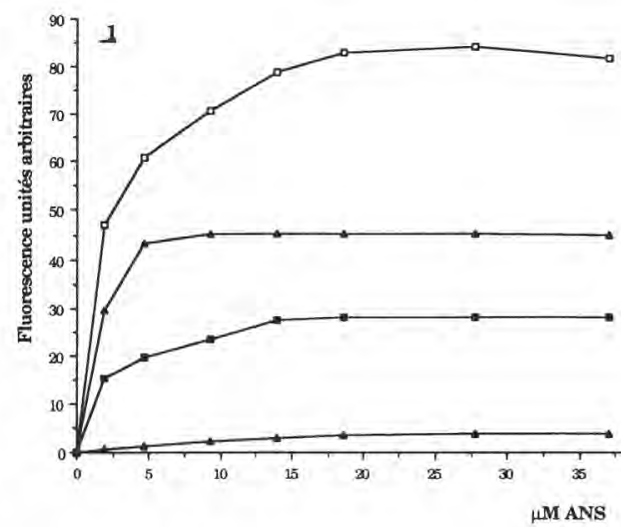

Fig 1. Variation de fluorescence des protéines natives du lactosérum $(4,5 \mathrm{mg} / \mathrm{l})$ avec des concentrations croissantes d'ANS en milieu tampon phosphate de potassium $50 \mathrm{mM}, \mathrm{pH} 6,80$.

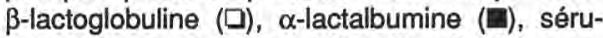
malbumine bovine $(\Delta)$ diluée 100 fois, immunoglobulines $\mathrm{G}(\mathbf{A})$.

Variation in native whey protein $(4.5 \mathrm{mg} / \mathrm{l})$ fluorescences related to the increasing ANS concentration in potassium phosphate buffer 50 $\mathrm{mM}, \mathrm{pH}$ 6.80. $\beta$-lactoglobulin (D), $\alpha$-lactalbumin (1); bovine serum albumin ( $\Delta$ ) diluted by 100; immunoglobulin $\mathrm{G}(\boldsymbol{\Delta})$.

L'hydrophobicité effective élevée de la BSA pourrait s'expliquer par l'existence de nombreux sites de fixation de composants hydrophobes, tels que les acides gras (Hsia et al, 1984). Ces sites de surface se-

Tableau I. Valeurs des paramètres de fluorescence obtenus pour les protéines purifiées de lactosérum $(4,5 \mathrm{mg} / \mathrm{l})$ en milieu tampon phosphate de potassium $50 \mathrm{mM}, \mathrm{pH} \mathrm{6,80.}$

Fluorescence parameters obtained for purified whey proteins $(4.5 \mathrm{mg} / \mathrm{l})$ in potassium phosphate buffer $50 \mathrm{mM}$, pH 6.80 .

\begin{tabular}{lccc} 
Protéines & $F_{\max }$ & $K_{f}(\mu M)$ & $F_{\max } / K_{f}$ \\
\hline$\beta$-Lactoglobuline & 86,2 & 1,55 & 55,6 \\
$\alpha$-Lactalbumine & 29 & 1,73 & 16,84 \\
Sérumalbumine bovine & 4866 & 1,12 & 4345 \\
Immunoglobulines G & 5,9 & 16,73 & 0,35
\end{tabular}


raient accessibles au solvant, donc à I'ANS.

En fonction de $K_{f}$, on distingue deux groupes de protéines; d'une part les protéines globulaires qui affichent des $K_{f}$ similaires traduisant une même affinité pour l'ANS, et d'autre part les IgG qui ont un $K_{f}$ 10 fois plus grand et un $F_{\max }$ faible. $\mathrm{Ce}$ dernier est à rapprocher de celui des caséines $\alpha_{\mathrm{S}}$ et $\beta$ observé par Shimizu et al (1985). En effet les caséines, tout comme les IgG, ont une structure flexible non globulaire, et présentent peu de sites de fixation pour les fluorophores hydrophobes. II semblerait donc exister une relation entre la structure tertiaire d'une protéine et son affinité pour l'ANS.

Le rapport $F_{\max } / K_{f}$, de par sa grande variabilité d'une protéine à l'autre, constitue un paramètre spécifique à chaque protéine.

\section{Mesure de l'hydrophobicité de surface sur des lactosérums industriels}

Nous avons effectué les mêmes mesures d'hydrophobicité que précédemment sur des lactosérums industriels.

La figure 2 a résume les variations de fluorescence sur des lactosérums préconcentrés doux (moyennes de 10 résultats pour chacun d'eux).

Les lactosérums préconcentrés de pâtes pressées cuites (PPC), où le lait n'a pas été pasteurisé, se caractérisent par une faible fluorescence comparée à celle observée pour les mélanges industriels de pâtes molles (PM) et pâtes pressées non cuites (PPNC), dont le lait a eu une pasteurisation haute (supérieure à $75^{\circ} \mathrm{C}$, 20 s). Quant aux lactosérums préconcentrés de grands mélanges PPC et PM, leur hydrophobicité de surface est intermédiaire.
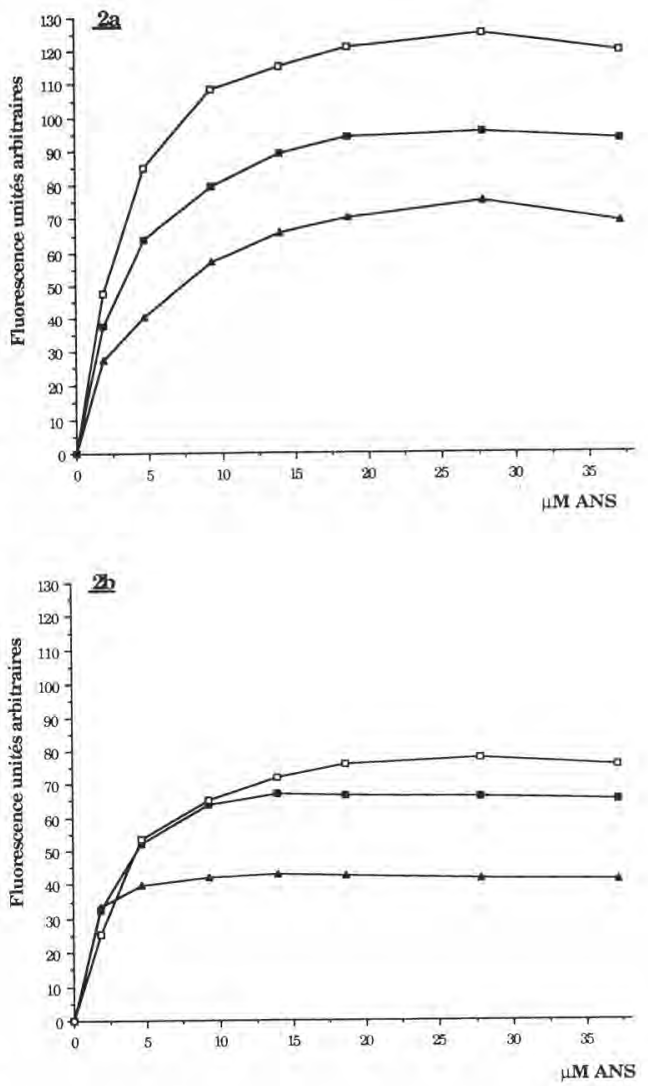

Fig 2. Variation de fluorescence en fonction de la concentration en ANS sur des lactosérums préconcentrés doux (a) et sur des lactosérums acides (b) en milieu tampon phosphate de potassium $50 \mathrm{mM}, \mathrm{pH} 6,80$, à des concentrations en protéines de $4,5 \mathrm{mg} / \mathrm{l}$. a: pâte pressée cuite ( $\Delta)$, pâte molle et pâte pressée cuite (四), pâte molle et pâte pressée non cuite ( $\square$ ); b: pâte molle au lait cru (A), pâte fraîche $(\mathbf{E})$, caséine préconcentrée ( $\square$ ).

Variation of preconcentrated sweet whey (a) and acid whey (b) fluorescences as a function of ANS in potassium phosphate buffer $50 \mathrm{mM}, \mathrm{pH}$ 6.80 with a protein concentration of $4.5 \mathrm{mg} / \mathrm{l}$. a: cooked pressed curd ( $\mathbf{\Delta})$, moulded curd and cooked pressed curd ( $\boldsymbol{\square})$; moulded curd and uncooked pressed curd (口); $b$ : raw-milk moulded curd ( $\mathbf{\Delta})$; fresh curd ( $\mathbf{(})$; preconcentrated casein (a). 
L'ensemble de ces lactosérums a subi le même traitement de préconcentration (température, extrait sec final); les seules variations observées proviennent de leurs origines fromagères.

Les variations de fluorescence observées sur les lactosérums acides de différentes technologies (moyennes de 10 échantillons pour chacunes d'elles) sont reportées en figure $2 \mathrm{~b}$. Les lactosérums acides de technologies pâtes molles au lait cru ont l'hydrophobicité de surface la plus faible. À l'inverse, ceux issus de technologies pâtes fraîches industrielles (PF), où le lait a subi une pasteurisation haute, se caractérisent par une fluorescence beaucoup plus importante, qui reflète une dénaturation protéique plus prononcée. Les lactosérums acides de caséinerie ont la fluorescence la plus élevée. Mais il faut rappeler que ces derniers ont été analysés sous forme préconcentrée; ils ont donc subi 2 traitements thermiques (pasteurisation du lait et préconcentration du lactosérum).

Les $K_{f}$ des lactosérums étudiés sont constants $(4 \pm 0,2)$, ce qui se traduit par une même affinité vis-à-vis de l'ANS. Le rapport $F_{\text {max }} / K_{\mathrm{f}}$ ne varie qu'en fonction de $F_{\max }$, et ne sera donc pas représenté graphiquement. Toutefois, dans notre étude, la valeur des $K_{\mathrm{f}}$ est le double des $K_{\mathrm{f}}$ observés pour les protéines globulaires natives du lactosérum. La fixation d'ions calcium par l' $\alpha$ LA pourrait en être une des causes (Musci et Berliner, 1985). Plus généralement, les protéines des lactosérums sont dans un environnement ionique complexe, ce qui peut modifier la valeur de la constante de dissociation par rapport aux protéines purifiées.

Pour les deux groupes de lactosérums (acides et doux) étudiés, se manifestent d'importantes variations d'hydrophobicité de surface. Elles traduisent une dénaturation protéique plus ou moins prononcée, mise en évidence par une augmentation de la fluorescence. Selon Mills et Creamer (1975), il existe pour la $\beta L G$ une région apolaire enfouie dans la structure tertiaire de la molécule, qui n'est donc pas accessible au solvant, donc à I'ANS. Cet environnement hydrophobe contient en outre deux tryptophanes qui se retrouvent en surface de la molécule après une dénaturation thermique. Cette région hydrophobe fixe alors l'ANS, et il en résulte une augmentation de fluorescence. Un mécanisme analogue semble être observé lorsque la BSA est soumise à un foisonnement (Clark et al, 1988) et qu'elle se déplisse à l'interface eau/air.

Les causes de ces dénaturations protéiques sont dues aux traitements thermiques infligés au lait, et par conséquent au lactosérum. Les premières protéines à se dénaturer thermiquement selon Cheftel et Lorient (1982) sont les IgG (instables à une température supérieure à $60^{\circ} \mathrm{C}$ ) puis la BSA $\left(65^{\circ} \mathrm{C}\right)$, la $\beta L G\left(75^{\circ} \mathrm{C}\right)$ et l' $\alpha \mathrm{LA}$ $\left(95^{\circ} \mathrm{C}\right)$. Mais vu les faibles proportions d'lgG (12\%) et de BSA (5\%) dans le lactosérum (Alais, 1984), nous pouvons admettre que les protéines impliquées sont essentiellement la $\beta \mathrm{LG}$ et, à un degré moindre, l' $\alpha$ LA.

De cette étude, il ressort que nous pouvons classer les lactosérums par la valeur de $F_{\max }$, selon leurs origines technologiques (fig 3a et 3b). Pagliarini et al (1990) ont réalisé un travail similaire avec différents laits commerciaux. Ces auteurs classent les laits en quatre groupes, selon leur hydrophobicité de surface. Elle augmente dans le sens lait cru, lait pasteurisé, lait UHT, puis chute brusquement pour le lait stérilisé en bouteille. Dans le lait cru, les groupes hydrophobes sont masqués dans la structure native des protéines, ils sont peu accessibles à l'ANS, ce qui se traduit par une faible fluorescence. Lorsque le traitement thermique augmente (lait pas- 
teurisé, lait UHT), il en résulte un déplissement des protéines. Les sites hydrophobes deviennent plus accessibles. La fluorescence croît donc en fonction de la sévérité
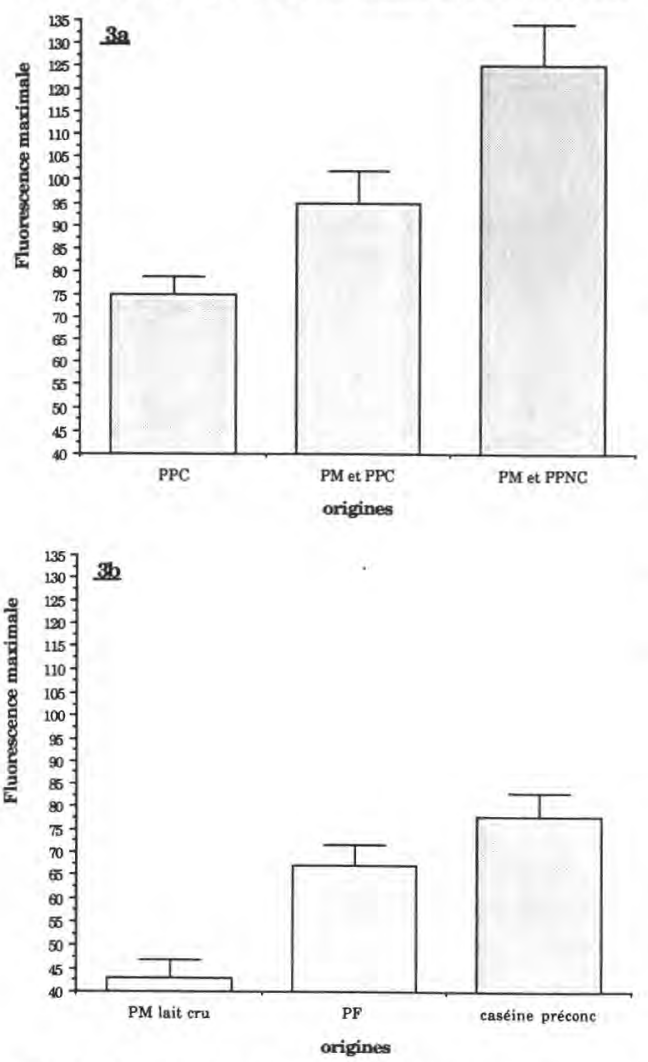

Fig 3. Évolution de la fluorescence maximale selon l'origine des lactosérums préconcentrés doux (a) et des lactosérums acides (b). a: pâte pressée cuite (PPC), pâte molle et pâte pressée cuite (PM et PPC), pâte molle et pâte pressée non cuite (PM et PPNC); $b$ : pâte molle au lait cru (PM lait cru), pâte fraîche (PF), caséine préconcentrée (caséine préconc).

Maximum fluorescence evolution according to sweet preconcentrated whey (fig 3 a) and acid whey origins. a: cooked pressed curd (PPC); moulded curd and cooked pressed curd (PM and $P P C)$; moulded curd and uncooked pressed curd (PM and PPNC). b: raw-milk moulded curd (PM lait cru); fresh curd (PF); preconcentrated casein (caséine preconc). des traitements thermiques. Finalement, lors d'une stérilisation $\left(120^{\circ} \mathrm{C}, 20 \mathrm{~min}\right)$, les protéines s'assemblent par interactions hydrophobes en agrégats rendant de moins en moins de sites accessibles à l'ANS, ce qui se traduit par une diminution de fluorescence (Bonomi et al, 1988). Néanmoins, nous n'avons pas observé de phénomène d'agrégation, dans nos lactosérums, qui pourrait gêner nos interprétations, car ceux-ci n'ont pas subi de traitements thermiques aussi drastiques que les laits stérilisés en bouteille.

La mesure de la dénaturation des protéines par cette technique est donc tout à fait appropriée à nos types de lactosérums. Pour parfaire l'étude, il est envisagé de corréler cette méthode à la détermination de la solubilité protéique au $\mathrm{pH}$ isoélectrique, afin de comparer ces deux techniques d'estimation de la dénaturation des protéines.

\section{CONCLUSION}

La spectrofluorimétrie utilisée pour estimer le degré de dénaturation protéique dans les lactosérums industriels est une technique facile à mettre en œuvre. La mesure de la densité optique à $280 \mathrm{~nm}$ suffit à ajuster la concentration protéique des échantillons. Même s'il s'agit d'une évaluation semi-quantitative (en unités arbitraires), la mesure par spectrofluorimétrie permet de faire un tri entre les différents lactosérums industriels, en fonction des traitements thermiques subis. Cette méthode rapide et fiable donne des indications précieuses sur le passé fromager du lactosérum qui nous permettent de comprendre le comportement évaporatoire du produit. En effet, l'évaporation est plus difficile à réaliser avec un lactosérum dont les protéines ont été dénaturées. 


\section{REMERCIEMENTS}

Ce travail a été réalisé grâce à une aide de la Mission Recherche de la région Lorraine.

\section{RÉFÉRENCES}

Alais C (1984) Science du lait. Sepaic, Paris

Bonomi F, lametti S, Pagliarini E, Peri C (1988) A spectrofluorimetric approach to the estimation of the surface hydrophobicity modifications in milk proteins upon thermal treatment. Milchwissenschaft 43, 281-285

Cheftel JC, Lorient D (1982) Les propriétés fonctionnelles des protéines laitières et leur amélioration. Lait 62, 435-483

Clark D, Smith L, Wilson D (1988) A spectrofluorimetric study of the conformational properties of foamed bovine serum albumin. $J$ Colloid Interface Sci 121, 136-147

Halpin M, Richardson T (1985) Selected functionality changes of $\beta$-lactoglobulin upon esterification of side chain carboxyl groups. $J$ Dairy Sci 68, 3189-3198

Hayakawa S, Nakai S (1985) Contribution of hydrophobicity, net charge and sulfhydryl groups to thermal properties of ovalbumin. Can Inst Food Sci Technol J 18, 290-295

Hsia JC, Wong LT, Feldhoff R (1984) Bovine serum albumin: Characterization of a fatty acid binding site on the $\mathrm{N}$-terminal peptic fragment using a new spint label. Biochemistry 23, 5930-5932

Kato A, Fujimoto K, Matsudomi N, Kobayaski $\mathrm{H}$ (1986) Protein flexibility and functional properties of heat-denatured ovalbumin and lysozyme. Agric Biol Chem 50, 417-420

Mills OE, Creamer LK (1975) A conformational change in bovine $\beta$-lactoglobulin at low $\mathrm{pH}$. Biochim Biophys Acta 379, 618-626
Mottar J, Bassier A, Joniau M, Baert J (1989) Effect of heat-induced association of whey proteins and casein micelles on yogurt texture. $J$ Dairy Sci 72, 2247-2256

Musci G, Berliner LJ (1985) Probing different conformational states of bovine $\alpha$ lactalbumin: fluorescence study with bisANS. Biochemistry 24, 3852-3856

Nakai S, Ho L, Helbig $H$, Kato A, Tung MA (1980) Relationship between hydrophobicity and emulsifying properties of some plant proteins. Can Inst Food Sci Technol J 13, 23-27

Pâquet D, Nejjar Y, Linden G (1988) Study of a hydrophobic protein fraction isolated from milk proteose-peptone. J Dairy Sci 71, 14641471

Pagliarini E, lametti S, Peri C, Bonomi F (1990) An analytical approach to the evaluation of heat damage in commercial milks. J Dairy Sci $73,41-44$

Paulson AT, Tung MA (1987) Solubility, hydrophobicity and net charge of succinylated canola protein isolate. J Food Sci 50, 15571561

Peri C, Pagliarini E, lametti S, Bonomi F (1990) A study of surface hydrophobicity of milk proteins during enzymatic coagulation and curd hardening. J Dairy Res 57, 101-108

Shimizu M, Saito M, Yamauchi K (1985) Hydrophobicity and emulsifying activity of milk proteins. Agric Biol Chem 50, 791-792

Slattery CW, Sood SM, Chang P (1989) Hydrophobic interactions in human casein micelle formation: $\beta$-casein aggregation. J Dairy Res $56,427-433$

Waniska RD, Kinsella JE (1984) Physicochemical properties of maltosyl and glucosaminyl derivates of $\beta$-lactoglobulin. Int J Pept Protein Res 23, 467-476 